\title{
Visual orientation by motion-produced blur patterns: Detection of divergence
}

\author{
T. L. HARRINGTON, M. K. HARRINGTON, C. A. WILKINS, and Y. O. KOH \\ University of Nevada, Reno, Nevada 89557
}

\begin{abstract}
Blur patterns are physiological "streaks" of photochemical and neural activity that occur whenever an observer and his visual environment are in relative motion. When retinal velocities are high, the impression of visual "flow" gives way to one of a field of "blur lines" whose patterns are rich with information about the motions and the optical textures that produced them. Simulated blur patterns were produced and thresholds measured for the detection of divergence at nine retinal loci. Sensitivity was somewhat greater in the central retina. Thresholds remained the same despite variations in pattern velocity, number of elements, and the presence or absence of an internal velocity gradient. Observers were able to orient abovethreshold patterns, but consistently underestimated the amount of slant.
\end{abstract}

In the usual conceptions of visual orientation by a moving observer, textural elements provide the same visual stimulation as they do under conditions of static viewing. Actually, texture in motion loses many of its static characteristics but gains important new characteristics as direct consequences of the motion itself and of the nature of the visual system. Specifically, points of light and dark in the static domain are transformed into streaks called motion bands or blur lines in the motion domain. To the visual system, a stationary line is a simultaneously stimulated row of retinal points, but, because of temporal "slack" in the photoreceptors and in the succeeding neural elements and their interconnection, perfect simultaneity is not necessary for a line to be perceived. As a result, when a single point of light or dark moving across the retina stimulates successive locations quickly enough, the visual impression is given of a line dragging along behing the point. Some factors controlling the length of this blur line are the velocity of the point, its intensity, contrast, hue, and context, and the state of the dark adaptation of the eye. The slope of the blur line will, of course, depend on the direction of movement of the point on the retina.

Thus, a textured field, to a moving observer's visual system, becomes a field of variously sloped and curved blur lines. The motion-related information in these "blur patterns" is not only in terms of motion of textural elements in time per se. Rather, by virtue of its characteristic shape, each blur line in the blur pattern displays at each moment the motion history of the element that produced it, and

This research was supported by Office of Naval Research Grant n00014-76-C-0398. Pilot work was supported by the Research Advisory Board of the University of Nevada, Reno. Requests for reprints should be sent to Thomas Harrington, Department of Psychology, University of Nevada, Reno, Nevada 89557. the observer is presented a time-varying stabilized image of variously curved and diverged stationary "lines." Figure 1 shows some typical naturally occurring blur patterns photographed from a vehicle moving at high speed.

Blur patterns are not restricted to high-velocity situations and may be important in all but the very slowest of visual transformations. The work of Brown (1931b) and Smith (1969), combined with the authors' observations, indicates that, even with angular velocities of motion less than $10 \mathrm{deg} / \mathrm{sec}$, substantial blur patterns can be formed. Thus, even at walking speeds a large portion, and perhaps all, of the visual field may be motion-blurred.

Despite the ubiquity of motion-produced "blur patterns," little research has been done on human processing of the information they convey. Yet such research has significance for theories of motion and form perception, as well as for the problem of maximizing human visual performance in highvelocity situations.

Two important blur-pattern parameters are the divergence and the curvature of the blur lines. The lines of a blur pattern diverge or converge differently in different parts of the field of view, providing orientation information, and they also diverge and curve in specific ways during altitude changes, accelerations, and changes of observer-texture relations. Divergence is defined here, in terms of the simulated blur-pattern displays used, as the angle between the outermost lines possible in the display (Figure 2).

Previous investigations (Harrington, 1967) have shown that human observers are sensitive to disparities of slope and curvature in fields of stationary line elements, and this study has demonstrated sensitivity to divergence when it occurred in motionproduced blur patterns. 

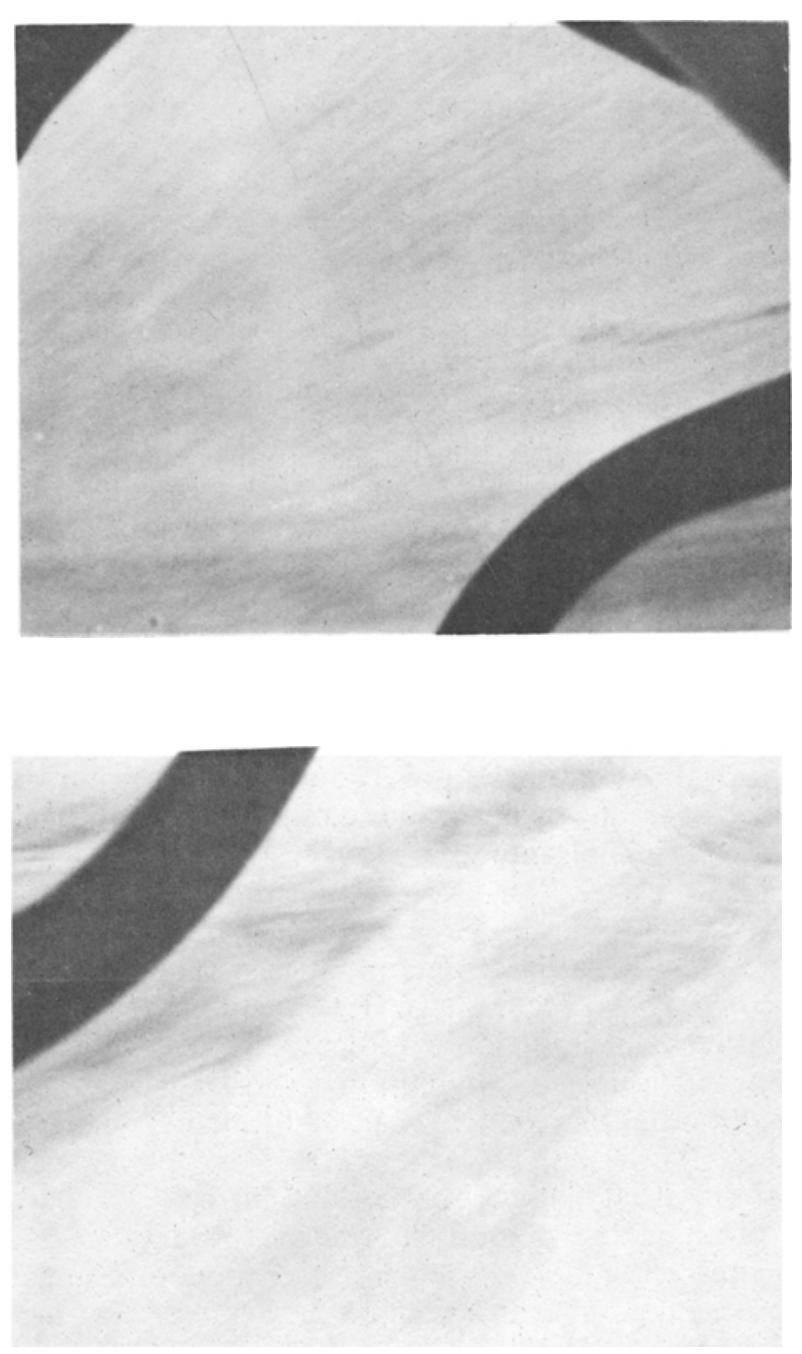

Figure 1. The upper photo, showing divergence, was taken to the front shortly before takeoff; the lower, showing divergence and curvature, was taken during landing.

\section{Low-Velocity Transformations as Stimuli}

Since the research to be described deals with a new area of visual perception, a survey of several related areas is provided. Optical transformations have recently received attention as sources of information about movement, form, and depth. Gibson (1950a) argued that transformation information, the orderly change of the optic array over time, serves as visual stimulation. A number of studies (Fieandt \& Gibson, 1959; Gibson \& Carel, 1952; Gibson, Gibson, Smith, \& Flock, 1959; Jansson \& Johansson, 1973; Jansson \& Runeson, 1977; Johansson, 1964; Johansson \& Jansson, 1968; Wallach \& O'Connell, 1953; Marmolin, Note 1) have asked which transformations are involved in motion and form perception. Johannson (1964) and his group have searched for the "decoding principles" by which the proximal array transformations are decoded and depth-related perceptions are given. Jansson (1977), Jansson and Johannson (1973), and Jansson and Runeson (1977) have attempted to specify a hierarchy of perceptual preferences for seeing rigid rotation, bending motion, and stretching motion in moving two-dimensional pattern transformations. Representative of these and other investigators of slow rotational transformations of forms, Braunstein $(1960,1962,1966,1977)$ has studied polar and parallel projections of the motion of rectangles and trapezoids. He proposed that angle change between the horizontal and vertical contours was the principal indicator of direction of rotation, with the difference in acceleration in the vertical playing a secondary role (1977). Von Hofsten (Note 2) has investigated proximal velocity functions as possibly being superstimuli that give depth information directly in the optic array. He has done so by moving a single dot according to particular functions. With Borjesson (Borjesson \& Von Hofsten, 1975, 1977), he has also formulated and tested a vector model of perception of translational and rotational transformations. In the same vein, Gibson (1950a) and Llewellyn (1971) have investigated pure optical expansion, or looming, as a possible primary stimulus for translatory motion in depth. Yet, in the search for purely optic transformations that are specifically related to form and depth perception,

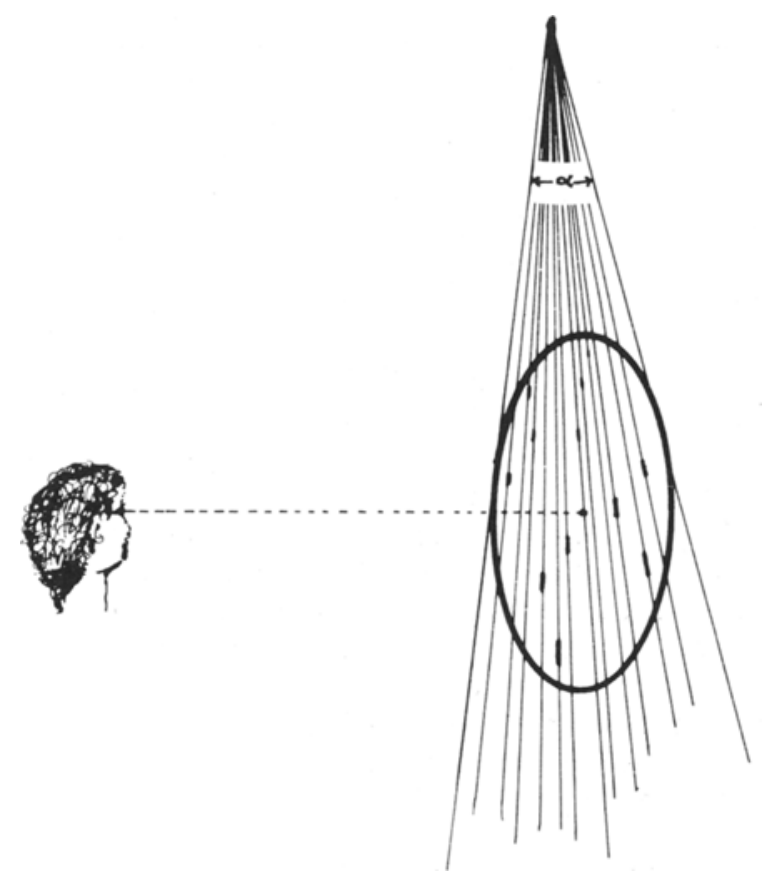

Figure 2. The divergence angle, alpha, is defined as the angle between the extrememost possible blur-line trajectories at the left and at the right of the display. Divergence so defined depends upon the size of the display. The situation is analogous to a moving observer's view through a porthole in his craft. 
the sizable realm of fast-transformation space perception is unexplored.

\section{The Effect of Fast Transformations on an Optical Array}

As higher velocities of the retinal image lead to motion-produced blurring, the domain of points, lines, and relationships encountered at low velocities is mapped into a new domain. To illustrate, consider the following mappings, from the slow-motion domain to the fast-motion domain, of certain elements and transformations usually thought to carry significant perceptual information: (1) Points are mapped into "lines," fine textures on the ground become blurred into fields of blur lines, and texture gradients become blur pattern gradients. (2) Lines are mapped into motion bands or elongated surfaces, whose widths depend on the length of the line and on its orientation. (3) Surfaces remain surfaces but are stretched out in the direction of motion to an extent depending on velocity, the amount and kind of light reflected, state of retinal adaptation, etc. (4) Brightness and velocity of a moving element are mapped into blur-line length because a very bright moving object will have a more lasting effect at each retinal locus. (Of course, state of dark adaptation, color, and other variables are again important.) (5) A shape's dimensions along the direction of motion are distended and are related to lightness of the resulting motion bands. The corners of a shape become blur lines, and the sides, motion bands. For instance, if a triangle were mapped through the transformation caused by blurring, one would see three blur lines, corresponding to the corners (unless two corners were moving in line with each other), and a configuration of motion bands, corresponding to the sides with possible overlapping, which would depend on the figure's orientation. (6) Motion parallax, the differential angular velocities of objects at different distances from the observer, now begins to involve blurline lengths because near objects will have higher angular velocities and thus will show greater blurring than distant objects. Also, a new kind of motion parallax emerges in which blur lines may "move" in relation to one another. (7) Motion perspective, apparent moving of elements along specific paths that depend on direction of movement and distance of the element from the observer, reverts to a situation like the linear perspective one finds in a completely static situation because the apparent paths of movement of the elements become blur lines that converge at the horizon, just as the railroad tracks and sides of squares do in the static situation. Because of this, both the divergence and the curvature in blur patterns become very important sources of motion information for an observer.
(8) Other perceptual information also changes during fast motion. For example, eye-head feedback from muscles, and depth cues involving eye-muscle change such as convergence or accommodation, shift their roles because the elements may be moving too fast for feedback to operate; in the blurring domain, it would still be possible, for example, to accommodate to produce maximally sharp contours of the blur lines themselves and thus form the basis for a judgment.

To illustrate the radical changes of the visual system's processing tasks when the foregoing blur transformations take place, consider a typical and common optical transformation, 'looming," which is itself transformed to a new entity in the blurring domain by motion. "Looming" is the perceived expansion of textural elements about some point as an object nears an observer or as the observer moves toward or over a surface (Schiff, Caviness, \& Gibson, 1962); even young animals respond to a pattern that looms rapidly. Several proposals have been made about how it might play a role in guiding locomotion. Gibson (1950a) at first speculated that the center of expansion in the looming pattern was maintained as the reference point toward which individuals wished to move. Llewellyn (1971) contended that subjects processed the pattern drift motions caused by offcourse movements and moved to nullify the drift. Gordon and Michaels (1965) argued that observers might use the locus of moving points that had zero transverse angular velocity. To a driver, for example, this would be the locus of points that appeared to be coming straight below, called the "locomotor flow line" by Lee and Lishman (1977). Calvert (1954) (in tacit allusion to the blur domain) has noted that operators of moving vehicles may use peripheral vision to analyze "streamer" information from motionblurred objects like road signs for guidance.

If the transformation of looming is now regarded as a blur pattern in the high-velocity domain, fast motion maps the textural points into blur lines. Gibson's task of finding the center of the expanding pattern, which Llewellyn found to be quite difficult for observers, becomes an easy visual task of finding the origin of a set of radial "lines." Llewellyn's "pattern drift" processing can be accomplished as a slope-of-"lines"-element task, at which the visual system is excellent. Gordon and Michael's locus of zero transverse angular velocity becomes the only "blur line" on the highway in front of the observer that is pointing directly toward him and passing directly beneath him.

At high velocities, then, perception of locomotion parameters could be facilitated by processing transformations of divergence, curvature, and blur-line densities, rather than the transformation of movingpoint positions. This would be true not only in the 
special case of expansion transformations, but also in the case of other transformations of relative textural position, such as the apparent "rotational" transformation of texture beside a moving vehicle when eyes maintain fixation on some point, or such as the translation of patterns to the side of a vehicle when the eyes merely sweep the passing array without fixation. Previous investigations (Harrington \& Sprenger, Note 3), using motion pictures with moving dots whose velocity functions were calculated to correspond to movement in various conditions, for example, fast looming, have shown that simulated blur-pattern divergence and curvature give three-dimensional perceptions from two-dimensional arrays. Also, the slopes of real blur lines relative to reference edges on an automobile, like the bottom of the side window, could be used as information for guiding an automobile. The blur patterns directly to the front of a speeding automobile quite sensitively indicated changes in the state of motion to observers, for example, by way of changes in curvature or by the blur-pattern orientation.

\section{The Effectiveness of Eye Movements in Minimizing Motion Blurring}

In a slow-motion situation, the moving observer will ordinarily use eye movements to help minimize motion blurring. His fixations allow him to sample information selectively from either a blurred array or from a retinally semistationary one, controlling the kind of information reaching different areas of the retina. But eye movements are only minimally effective in minimizing blurring, especially at high angular velocity, because a moving observer has, under normal circumstances, a single stationary point in his retinal image. Gibson, Olum, and Rosenblatt (1955), using Cartesian coordinates, Gordon and Michaels (1965), using spherical coordinates, and Whiteside and Samuel (1970), using a torroidal coordinate scheme, have derived formulae for calculating the angular velocities of different positions surrounding a moving observer. Their work shows the existence of a locus of nonfixated points that do have zero relative angular velocity at a given moment when an observer maintains fixation on a single point; these points are not stationary in the usual sense, but are momentary pauses before reversing apparent direction and, in the next moment, they will accelerate and blur, and new points will pass through the circular (if the eye is at ground level) zero-velocity locus.

Even though eye movements cannot provide a stationary image in the usual sense, an observer in motion does profit greatly from moving his eyes. Accordingly, much research has been devoted to specifying positioning and eye-movement pursuit parameters (Ludvigh, 1952; Rashbass, 1961; Westheimer, 1954; Yarbus, 1967). It is evident that the foveal image can be rendered semimotionless at least for short periods. Since large amounts of visual information can be buffered and processed after brief "stationary" visual presentations (Averback \& Sperling, 1961; Sperling, 1960; and others), at low angular velocities an image on the retina can provide good visual information about form and slow relative movement. Cohen and Studach (1977) have studied fixation behavior of automobile drivers and found mean fixation times on the order of only half a second, implying fairly efficient sampling.

\section{Failure of Eye Positioning: When Blurring Begins}

It has been well documented that the dynamic visual acuity (DVA) of a moving observer decreases as relative angular velocity increases, even though the eyes attempt to pursue the target. According to Ludvigh (1948), even when the eye is permitted to move in an effort to follow a moving object, visual acuity deteriorates rapidly with increasing angular velocity of the object. With an observer's eyes pursuing at up to $50 \mathrm{deg} / \mathrm{sec}$, dynamic and static acuity are similar. Dynamic acuity begins to fail when either the observer or the object moves at about $50 \mathrm{deg} / \mathrm{sec}$ (Miller \& Ludvigh, 1962). According to Hulbert, Burg, Knoll, and Mathewson (1958), dynamic and static visual acuity of an observer with $20 / 20$ vision falls to $20 / 200$ when the observer is moving relatively at $110 \mathrm{deg} / \mathrm{sec}$ (Ludvigh \& Miller, 1958).

The eye is capable of very fast movements and could easily keep up with very high-velocity movement. So Miller and Ludvigh (1962) have reasoned that the decline in DVA is due to an inability of the eyepositioning system to match angular velocities well enough to provide a still image on the retina. Thus, blurring results. At tracking rates as low as $20 \mathrm{deg} / \mathrm{sec}$, the eye's angular velocity has been seen to vary sporadically, and at $110 \mathrm{deg} / \mathrm{sec}$, with the eye attempting "smooth pursuit," velocities of $250 \mathrm{deg} / \mathrm{sec}$ can be seen. It is known that the eye could keep up well enough with a moving object, for, on 40-deg movements during voluntary changes in fixation, angular velocities of $400 \mathrm{deg} / \mathrm{sec}$ have been measured. For 90-deg shifts, velocities can approach 1,000 deg/ sec (Haber \& Hershenson, 1973), and the average velocity of small movements has been estimated at between 100 and $200 \mathrm{deg} / \mathrm{sec}$ (Miller \& Ludvigh, 1962). Neither is the DVA decrement likely due to errors of the positioning mechanism that centers the target image off fovea, because off-fovea measurements of static acuity have shown that a 2-deg displacement produces an acuity decrement to only about 20/25 (Ludvigh, 1949).

\section{Failure of Contour Mechanisms: \\ Formation of Blur-Line Contours}

In the areas around a moving observer where 
angular velocity causes contour perception to be lost, potentially useful blur contours emerge. Cheatham (1952), employing a masking technique, showed that contour formation takes about $30-100 \mathrm{msec}$, and there is recent evidence from work on dynamic contour perception (DCP) (Smith \& Gullick, 1956) that clear contours fail to be formed at angular velocities as low as $14 \mathrm{deg} / \mathrm{sec}$.

The perception of visual contours begins to suffer probably because the positioning mechanisms of the eye do not afford the retina a stable image of sufficient duration. Smith and Gullick (1956), elaborating Michotte's (Note 4) observation, showed that the contours of a .5-deg black parallelogram moving across a 5-deg white field begin to blur at about $14 \mathrm{deg} / \mathrm{sec}$ (but if the square is seen to be stationary at the beginning and at the end of its excursion, then its contours remain clear at higher velocities). Smith and Gullick present data collected by increasing stationary pre- and postexposure time to $300 \mathrm{msec}$. At this value, the angular velocity at which contours could be seen clearly had increased (linearly) to around $24 \mathrm{deg} / \mathrm{sec}$. That vision begins to falter at this low value is surprising in view of the previously noted abundance of higher angular velocities in many visual situations. Yet, even though normal contour perception of a moving observer is impaired as relative angular velocity increases, blur lines can lengthen, forming a new kind of contour, the "contours" of the blur lines themselves. Retinal velocity, which diminishes conventional processing, may prove to be an asset in some situations.

\section{Characteristics of Motion-Produced Blur}

A full understanding of blur patterns requires the consideration of individual blur-line formation. Motion blurring occurs because the photochemical and the neural effects of visual stimulation do not stop immediately when the stimulus is removed. Perceptually, stimulation of the visual system seems to linger and reverberate. If a stationary point of light is presented to the retina, the corresponding perception of a trained observer is not simply a momentary flash, but rather a series of pulses of varying durations and brightness (McDougal, 1904-1905).

Similarly, when an image is moved smoothly across the retina, successively stimulating a sequence of points, observers see these pulses, possibly interacting with effects of the movement itself, as a series of blurred duplicate images moving along behind the original, following by a distance that is dependent on the speed of movement, according to McDougal. The most immediate of these images or pulses (Graham, 1934) are the so-called Charpentier's bands. Slower alterations (of brightness) succeeding the bands produce a series of positive afterimages of which the most prominent, the Purkinje afterimage, is absent or very faint in the center of vision (as would be expected if these are due to rod action). The more typical response from untrained moving observers, for example, automobile passengers, suggests that only streaks or "blur lines" are seen. This is substantiated by experimental observations. Segner, D'Arcy, and Cavallo (quoted in Helmholtz, 1925), probably among the first to investigate motionproduced blurring, whirled a glowing coal in a circle and, while noting the fiery trail that followed it, failed to mention reverberatory phenomena. DeSilva's (1929) account is representative of the reports of other workers that followed. He employed a laterally moving slit of light and, excluding pursuit movements of the eyes, noted the effects on perception of the slit at different angular velocities. As the angular velocity increased from 3 to $116 \mathrm{deg} / \mathrm{sec}$, the appearance progressed through six phenomenal steps: At the lower velocity, there was distinct contour; then outlines became slightly blurred, acquired luminous tails or afterglows, and became sheets of light unrolling at the beginning of the object excursion and rolling up at its termination; and finally, at $58-116 \mathrm{deg} / \mathrm{sec}$, the motion sheet filled the whole $2.7-\mathrm{cm}$ excursion and was somewhat vibratory, still showing the direction of movement; above this velocity, the sheet of motion became stationary in all respects. J. F. Brown (1931a) described the appearance of a black patch on a white ground as angular velocity increased, noting backward apparent movement in certain velocity ranges. Smith (1969) has recently measured velocity thresholds for DeSilva's later phases, where bands of motion are seen, and has found that higher velocities are required for circular "movement bands" if the arc length is increased or luminance is increased. She has found that the response in white light for a $25-\mathrm{cm}$ target (considerably larger than we use) at $5.7 \mathrm{deg}$ from the fovea is determined by a scotopic mechanism at all luminances and a photopic mechanism at high luminances, emphasizing the importance of retinal locus to blur-pattern perception. At very high velocities, invisibility sets in, the critical velocity depending on the target's angular velocity, on the state of adaptation, and on relative intensity of the target (Pollock, 1953). A theoretical explication of the temporal and spatial summative properties of the visual system in a similar context is available in von den Brink (1957).

\section{Static and Dynamic Perception of Surface Slant}

There is a small body of earlier research on the perception of slant or divergence. Gibson (1950a) initiated research in this area by determining that a texture density gradient could be a sufficient stimulus for the perception of a surface slant. Flock and Moscatelli (1964) and Phillips (1970) showed that, in the utilization of texture gradients, size and shape 
regularity were more important than regularity in distribution of elements. Clark, Smith, and Rabe (1956) demonstrated that contour convergence or divergence is much more effective than texture gradients in eliciting judgments of slant. Youngs (1976) found linear perspective a more effective cue than binocular disparity for judging slant. Form ratio, involving the relative shrinkage of certain contours in the figure with rotation, was also only secondarily useful when compared with convergence (Braunstein \& Payne, 1969) in the case of static displays; however, form ratio became a primary factor for dynamic perception when a slanted display was rotated. Judgments of slant involving either rotation or translation tended to be fairly close to displayed slant, while static judgments were usually underestimates, sometimes by as much as $50 \%$ perhaps, as Braunstein (1976) suggests, because of the presence of conflicting flatness cues in static displays.

\section{The Information in Patterns of Blur Lines}

Perceptually, the importance of motion blurring stems from the formation of blur patterns in which the individual moving elements appear as fields of blur lines variously sloped and curved in vision. Figure 3 shows some sketches representing the typical blur patterns that are readily visible directly

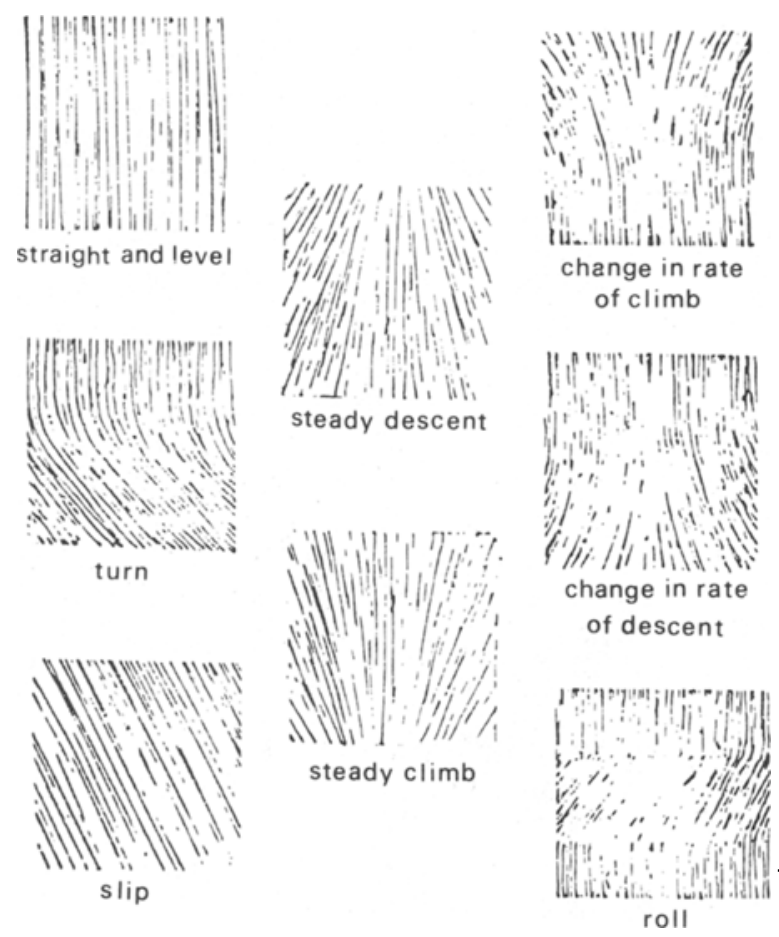

Figure 3. Blur-pattern correlates of attitudes of movement. below a moving observer, for example, a low-flying pilot, during basic types of motion. Here the visual system is dealing with fields of semiparallel curved or straight lines, not with moving points, as was previously noted. The apparent lengths of these fleeting blur lines are determined primarily by angular velocity, but also by the amount of reflected light, the wavelength, the state of dark adaptation, and the contrast (Harrington, Note 5). The relations between type of motion and blur-pattern change as illustrated in the figure are as follows: (1) rate of descent (ascent) is indicated by degree of blur-line vergence (convergence or divergence), and where an observer looks in his field of motion will change the blur pattern vergence he sees; (2) roll rate is indicated by relative blur-line slope, role acceleration by blur-line curvature; (3) amount of slip is indicated by relative blur-line slope; (4) change in yaw angle is indicated by blur-line curvature; and (5) rate of change of these is indicated by additional blur-line curvature superimposed on the appropriate basic pattern.

Work by Beck (1966a, 1966b, 1967), Harrington (1967), and Olson and Attneave (1970), using line elements, has shown that the human visual system is sensitive to the information carried by relative slope and curvature in fields of lines. In a search task in which a line segment of disparate slope was to be selected from a field of parallel line segments, the observer responded much more quickly to slope disparities than to attributes such as disparities in length. DiOrio (1979) has measured comparably low thresholds for divergence between pairs of static line elements that varied in separation, length, and exposure duration. Sulzer (1954) found comparably high sensitivity to divergence between drawn line elements.

\section{Determining Divergence Thresholds from Parallel}

In this study, thresholds for motion-produced divergence were determined for different parts of the visual field by using nine fixation points ranging from central (foveal viewing) to extremely peripheral ( $80 \mathrm{deg}$ visual angle to the side of the fovea). Displays moved at three velocities, slow $(20 \mathrm{deg} / \mathrm{sec})$, medium (40 deg/sec), and fast $(80 \mathrm{deg} / \mathrm{sec})$. The experiment also assessed the extent to which human observers could use divergence information for orientation. Two different methods of simulation were used to create the blur patterns-an optical method that produced "natural" patterns in which other variables existed along with divergence and an electronic method that produced "pure" divergence patterns. Finally, displays composed of only 16 elements per frame were compared with displays utilizing a finer texture with 32 elements per frame. 


\section{METHOD}

\section{Subjects}

Observers were students at the University of Nevada, with normal visual acuity, and were paid for their participation. Ten students were run for $15 \mathrm{~h}$ each; one eventually had to be eliminated because of fixation problems.

\section{Stimulus Materials}

The stimuli, presented either directly with a video monitor or with an oscilloscope face shown on the monitor, consisted of fields of randomly placed luminous line elements moving vertically from top to bottom. The patterns were of two types-the first, called natural or optically generated, having elements that increased in size and velocity as they moved down the display, and the second, called synthetic or electronically generated, having elements that retained constant size and constant velocity throughout their travel.

The patterns varied in the amount of vergence (divergence or convergence $-0,30,40,50,60$, or $70 \mathrm{deg}$ ). The term "vergence" refers here to both the vergence of blur lines and to the relative lateral motion, together or apart, of the elements. This experiment was not designed to separate these. Patterns had either 16 or 32 line elements per frame and stimulated any of nine separate fixation points on the retina. The elements comprising the blur lines moved at average velocities of 20,40 , or $80 \mathrm{deg} / \mathrm{sec}$, measured at the display center. These three velocities were chosen to represent the range found in naturally occurring blur patterns. The display subtended $12 \mathrm{~cm}$ at a viewing distance of $76 \mathrm{~cm}$.

\section{Apparatus for Stimulus Generation}

The natural stimulus patterns containing velocity and size gradients were prepared by videotaping moving $13-\mathrm{cm}$-wide belts of opaque photographic film from different angles to provide divergence. These were transported at appropriate velocities and backlighted by a photo-interpreter station. Moving line elements were areas of the film from which the emulsion had been removed to allow light to shine through. These were of lengths specific to each pattern velocity so that, for successive video frames, a given line element would advance exactly by its own length. This provided continuous successive spatial stimulation of the retina with no gap between line elements and with no overlapping. Actual element sizes for 20,40 , and $80 \mathrm{deg} / \mathrm{sec}$ were, respectively, .3, .6, and $1.3 \mathrm{~cm}$. The elements' widths were $.3 \mathrm{~cm}$.

Stimuli were ordered and assembled on a final videotape in a counterbalanced order so that each divergence value appeared nine times. Separate tapes were made for each of the three speeds.

A schematic diagram of the synthetic blur-pattern generation is shown in Figure 4. Sixteen vertical lines are swept and each is divided into 32 segments, only one of which is bright enough to see. This pattern is advanced at a variable rate. Electronically, the frequency of a central clock running at $32 \mathrm{kHz}$ is successively divided five times in the 32-step-per-line generator and four more times in the 16-line generator. These nine outputs address a memory containing one bit corresponding to an arbitrary predetermined locus on a vertical line for each line that is generated. The oscilloscope is swept vertically synchronously with the divide chain, and the output of the memory is amplified and used to modulate the Z-axis or brightness stage of the oscilloscope. Then, for each of the 16 vertical lines, one of the positions on it will be painted by the $\mathrm{Z}$ modulation, producing a random-appearing pattern of 16 elements. This pattern can be advanced at multiples of $1 / 60 \mathrm{sec}$ to produce a variable velocity of the pattern. Divergence is produced by adding a variable amount of the sweep signal to the horizontal displacement signal.

Patterns of 2-sec duration were prepared for each divergence angle. At the 80 - and $40-\mathrm{deg}$ pattern velocities, natural patterns were made with 32 line elements. At the $20-\mathrm{deg} / \mathrm{sec}$ velocity, patterns were constructed with both 32 (for the natural) and 16

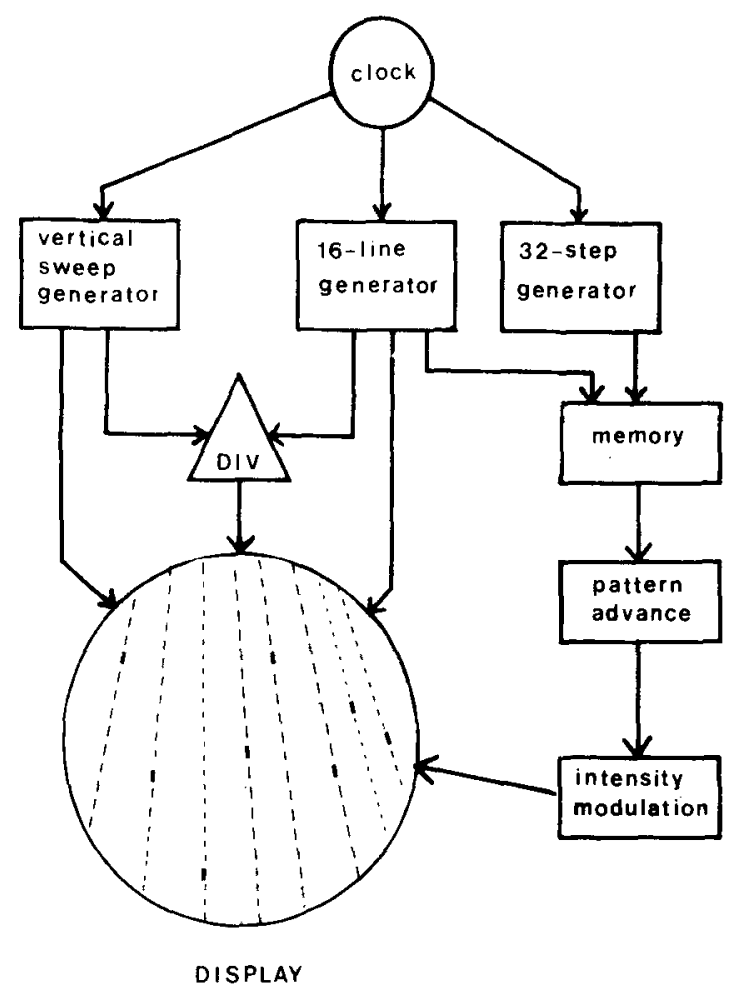

Figure 4. In common synchronization with the clock, vertical lines on the display are produced by the vertical-sweep generator, displaced successively from left to right by the 16-line generator, and modulated to produce one element per line by the 32-step generator, the memory and the intensity modulator. Divergence is produced by mixing varying amounts of sweep signal with the horizontal-displacement generator.

(for the synthetic) elements/frame to allow a comparison between the natural and the synthetic patterns, which always had 16 elements/ frame. Thus, each subject saw 945 unique stimulus configurations: 15 vergence values by 9 fixation points by 3 velocities for photointerpreter-generated patterns with 32 elements/frame, the same for the synthetic patterns with 32 elements/frame, and, in addition, 15 vergence values by 9 fixation points for photo-interpretergenerated patterns at $20 \mathrm{deg} / \mathrm{sec}$ with 16 elements/frame.

\section{Procedure}

Observers were run individually, with each observer seated in a semidarkened booth centered in front of a Conrac display monitor with a circular mask $12 \mathrm{~cm}$ in diameter. Earphones were worn to mask any noise outside the booth; a patch was worn over the left eye. During the first session, each observer was familiarized with the three velocities and the nine fixation points he was to employ. The fixation points were lighted letters in front of the black wall in which the monitor was embedded, all at equal distance from the eyes. The observer's eyes were monitored from outside the booth to be sure he maintained fixation despite the movement on the screen. One observer had to be eliminated for inability to maintain fixation.

During a trial, the observer fixated monocularly prior to each stimulus presentation, and afterward reported whether he had detected any convergence or divergence, gave a measure of the confidence in his judgment, and indicated the perceived plane of the blur lines by orienting a paddle connected to a protractor readout device. The sound track on the videotape was used to instruct and cue the observer. Each trial was $2 \mathrm{sec}$ in duration and the interstimulus interval was $6 \mathrm{sec}$. A single session lasted $1 \mathrm{~h}$. 


\section{RESULTS}

Figure 5 shows the threshold data pooled over convergence-divergence and over retinal loci for the three speeds. An analysis of variance was performed using a three-way factorial design (Convergence/ Divergence by Speed by Retinal Loci) with subjects as the blocking variable. There were no significant effects of direction of vergence or of speed. The effect of retinal locus on threshold judgment was highly significant $(\mathrm{p}<.001)$ as were the variations between observers $(p<.001)$. Figure 6 shows the resultant thresholds when the data are summed over speeds and also shows how the threshold rises with increasing distance from the center of the fovea. The "best" subject had vergence thresholds of $2 \mathrm{deg}$ or less for distances up to $40 \mathrm{deg}$ from the fovea, whereas the "worst" had thresholds around $10 \mathrm{deg}$.

A two-way factorial analysis of variance (Actual Slant by Retinal Locus) blocked on subjects was performed on the paddle-setting data; scores were the errors appearing on each trial in the observer's orientation of the surface readout device. Again, retinal locus of stimulation was highly significant $(p<.001)$, as were differences between observers $(p<.001)$; in addition, the actual slant of the displaced surface made a difference in how accurate the observer's judgments were. Additional comparisons were made using Tukey's HSD test. These indicated that errors were smallest when the orientation of the displayed surface was actually vertical, that is, when the blur lines were all parallel. Moreover, errors were significantly larger when the displayed surface was tilted with the top toward the subject (converging) than when it was tilted with the top away (diverging).

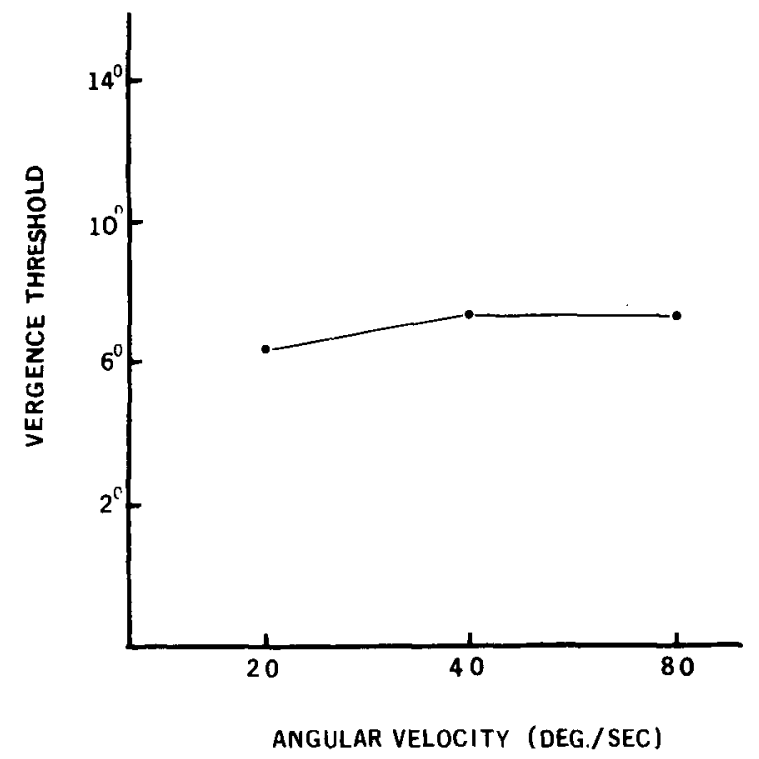

Figure 5. Vergence thresholds for the three pattern velocities pooled over convergence-divergence and retinal loci.

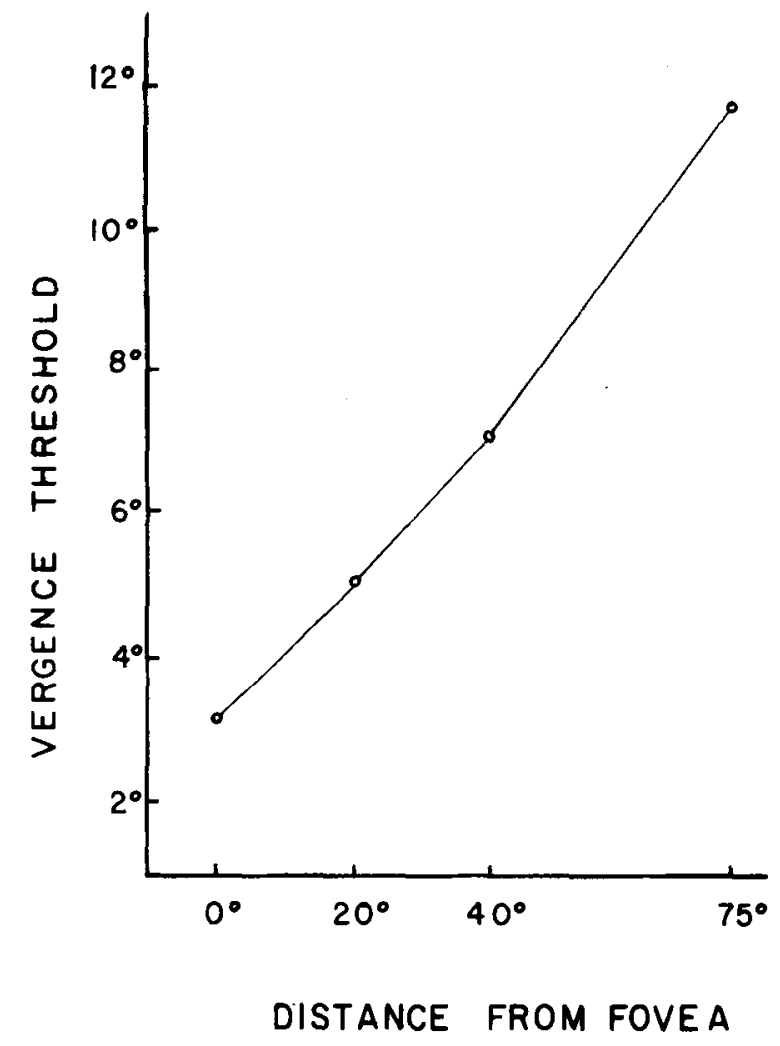

Figure 6. Threshold increases with distance from the fovea.

This may occur because of our greater experience with orienting in natural situations in which terrain rises in the distance. In addition, significantly larger errors were made when the display stimulated areas farther toward the periphery of the retina. This result is in keeping with the higher threshold values found for the same peripheral areas. Similarly, smaller errors were made in the cases of central viewing and viewing only $20 \mathrm{deg}$ above and below the central point. In terms of orientation accuracy, there were no significant differences between stimulation of the left and right halves of the visual field, or between stimulation of the top and bottom halves. There were no significant differences between the central point and $20 \mathrm{deg}$ above, $20 \mathrm{deg}$ below, and $40 \mathrm{deg}$ above, despite the fact that there were also no significant differences between the central point and $40 \mathrm{deg}$ left, $40 \mathrm{deg}$ right, $40 \mathrm{deg}$ below, and $40 \mathrm{deg}$ above. Performance to the left and right extremes was significantly poorer.

Figure 7 shows the mean paddle settings that observers selected for each of the displayed slants, as well as the perfect-performance line where settings conformed perfectly to displayed slant. There is greater accuracy when the elements are parallel, as noted above, as well as a tendency toward underestimating both with convergence and divergence. Figure 8 reveals how much difference there was in orientation accuracy between the most adept and the 
poorest processors of the information. A Spearman rank-order correlation coefficient between detection threshold and orientation error was $+.738(p<.05)$, suggesting that observers who were good at one task tended to be good at the other as well.

The results were analyzed for possible practice effects. The mean number of errors for the first three and last three sessions for each speed was computed for five of the subjects who had not participated in a short pilot study. A two-way factorial analysis of variance (Speed by First vs. Last Sessions) blocked on subjects was performed. The reduction in errors with practice was highly significant $(p<.001)$. The error rate did not vary significantly across speeds, nor was there any interaction between speed and amount of practice.

The effect of method of production was also analyzed. Stimuli differed in representing natural divergence or "pure" divergence and in being composed of either 16 or 32 elements. A two-way factorial analysis of variance (Retinal Location by Method of Production) was performed, with subjects as a blocking variable. Neither number of elements employed nor method of production, "optical" vs. electronic, had a significant effect on threshold values. Again, retinal locus of stimulation and differences between observers were significant $(p<.001)$.

Mean confidence ratings on a scale ranging from
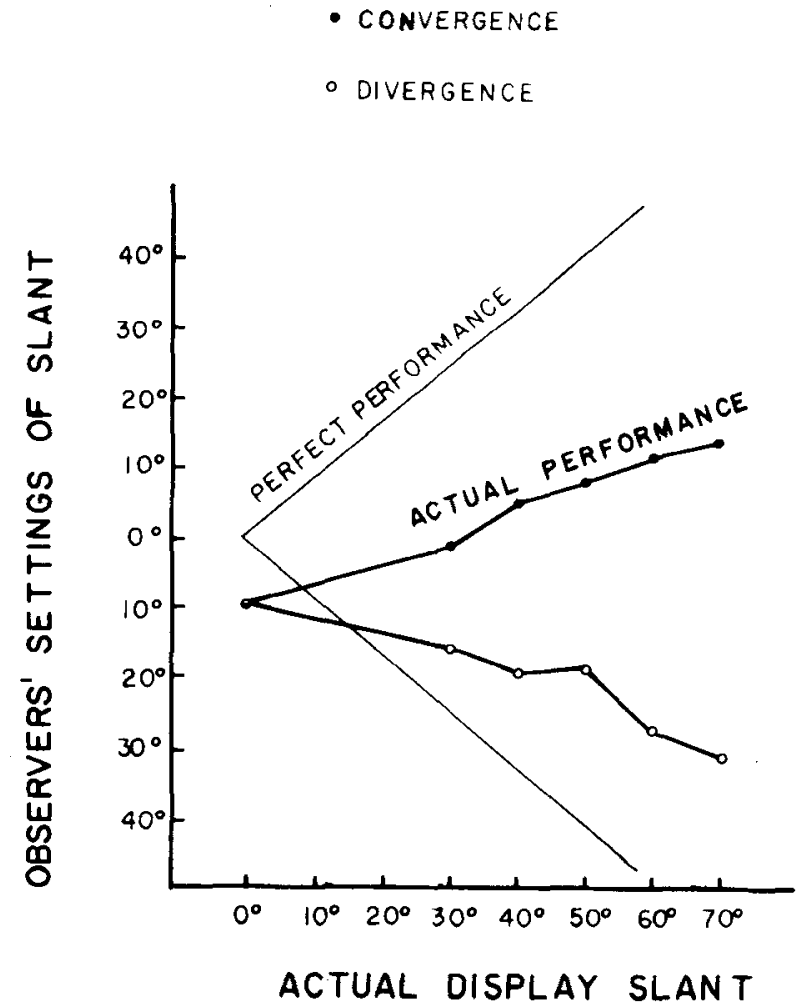

Figure 7. Observers' ability to use vergence for orientation.

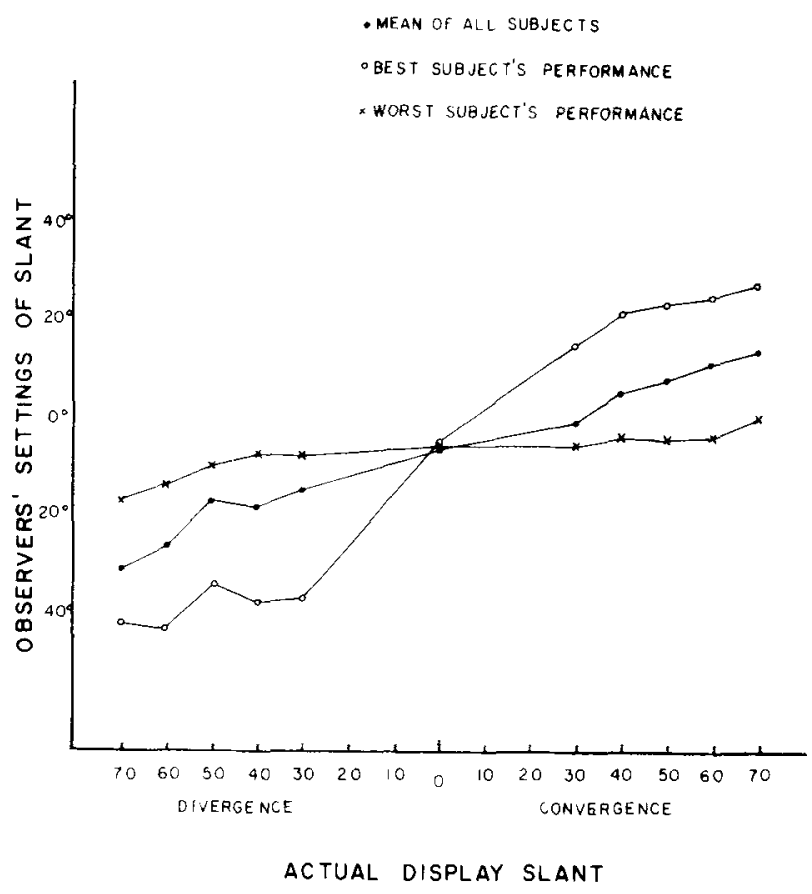

Figure 8. Differences in individual performances using vergence for orientation.

1 to 5 (where 5 represented a very high level of confidence) for vergence judgments at different fixation points were 3.93 for central viewing and 3.12, 3.04, and 2.23 , respectively, for 20,40 , and $75 \mathrm{deg}$.

\section{DISCUSSION}

This is the first in a series of investigations undertaken to determine whether humans are able to orient in depth using the various forms of information available in fast-motion-blurred visual patterns. This study dealt with pattern divergence information. The divergence in blurring patterns contains information for orientation, but the question of whether observers are sensitive enough to the variable to use it effectively has been previously unanswered. It was also unclear whether divergence in a moving pattern would be sufficient for producing percepts of depth, or whether the velocity gradients and element size gradients that accompany divergence in nature are necessary as well. It was not known if the important aspect of divergence was related to the motion of the pattern elements or to the forms of the blurs. There was no existing knowledge about the effect on divergence usability of pattern details like velocity, direction of motion, or element density, and the distribution of divergence sensitivity in the visual field was unknown.

\section{Observers}

Overall, observer sensitivity to blur-pattern diver- 


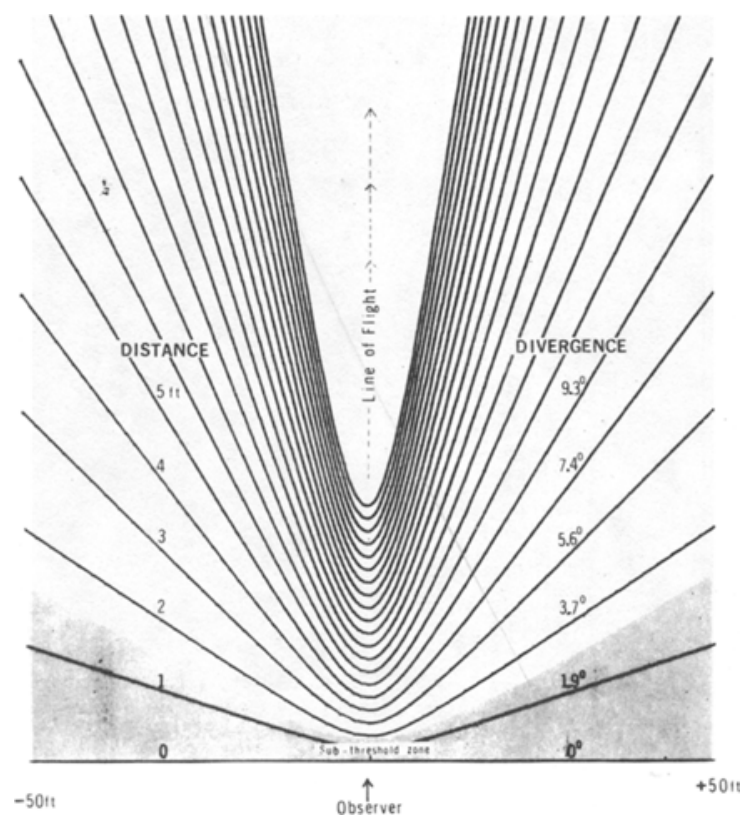

Figure 9. Isoverges, loci of equal vergence, to the fore of an observer moving straight and level with his eye $5.5 \mathrm{ft}$ above the horizontal plane in the direction indicated by the arrow. Distances are from the observer to the respective hyperbolas. The actual divergence angle that each isoverge represents is shown. For example, anywhere along the contour labeled $3 \mathrm{ft}$, the observer will see a divergence of $5.6 \mathrm{deg}$. All divergences below average foveal threshold, as determined by the experiments, lie approximately in the shaded zone.

gence was easily high enough to make it potentially useful for visual orientation. Figure 9 shows a typical zone of divergence blindness for the average observer. Even so, the variability between subjects was considerable. Certain subjects performed very accurately on both the threshold and the paddlesetting tasks, whereas others seemed relatively at a loss on both tasks and, during the experiments and debriefing, said they could not see a depth effect or divergence at all, or could see them only with extreme amounts of divergence. The impression was strong that there could be two types of observersthose weak, or even lacking altogether, in this type of visual processing, and those who are quite good at it. At least one observer in the weak category did have a history of visual orientation difficulty: She had reduced dynamic visual acuity while driving and difficulty in learning to assess the distance to the ground during the final phases of landing an airplane. Recent neurophysiological findings (Cynader \& Chernenko, 1976) and previous perceptual observations (Pantle \& Picciano, 1976) have shown the possibility of separate visual system processors for form, motion, and location. Perhaps observers are endowed differently in this regard.

\section{Retinal Locus}

Divergence sensitivity as a function of retinal locus was investigated to see whether a radical falloff from central viewing would be found as with acuity. The results of varying fixation points indicate that the most sensitive use of blur-pattern divergence with the displays that were used was possible with central viewing. However, reasonable peripheral sensitivity was evident.

In general, the closer to the fovea, the better is divergence detected. Fixation points that were closest to central viewing showed threshold values of 2-3 deg and were superior to the next closest to center with threshold values of $7.1 \mathrm{deg}$. Of the two farthest points, the nasal retinal showed a lower threshold than did the temporal, but both had higher thresholds than any other point.

\section{Display Velocity}

Blurred patterns such as were used here contain both form information generated by blurring and motion information from the element motion. When pattern velocity was doubled, the amount of form information remained phenomenally the same because blur-line length did not change. The lateral or divergent component of motion, however, was then doubled in velocity.

It would seem that, if the visual system were detecting motion aspects of divergence in the blur patterns, threshold would be changed when the motion parameters were. In the present experiment, three pattern velocities were used to determine tentatively the functional relationship. The experiment was merely to assess performance with subjects fixated to blur the pattern and was not specifically designed to decide on the relative importances of the form or the motion components of the divergence. However, the fact that thresholds were not affected by velocity hints at the possibly lesser importance of movement information from blur patterns.

Another experiment performed in this laboratory by DiOrio and Harrington (Note 6) was more directly designed to determine whether briefly presented form information alone would allow sensitive divergence thresholds. Using two-line patterns whose elements appeared and disappeared almost instantaneously with no motion, it was found that divergence threshold values were similar to those from the present experiment.

\section{Convergence-Divergence}

Patterns either diverged at the top, as would be seen during inverted flight, or at the bottom, as in normal locomotion over the ground. This condition was included because it was thought that the visual 
system might have an affinity for the type of pattern it nearly always receives. Such was not the case for divergence sensitivity.

\section{Number of Elements}

The comparison using optically generated blur patterns of 16 vs. 32 elements was not significant. It may be that, for the simpler aspects of blurpattern divergence processing, even fewer elements are needed. Still further experimentation is suggested to see if very fine textures with a great many elements might not carry blur-pattern information more effectively. Perhaps, in this context, the difference between 16 and 32 elements is small, although in some other conditions involving line elements with common slope, information is greatly enhanced by an increase of this magnitude in number of elements (Harrington, 1967).

\section{Natural vs. Synthetic Patterns}

Naturally occurring blur patterns have gradients of element velocity, element size, and element density, as well as vergence. The synthetic patterns electronically produced for these experiments have elements of unchanging velocity and differ from one trial to the next only in the amount of divergence between element trajectories. Element velocity (von Hofsten, Note 2), size change (Ittleson, 1951), and element density (Gibson, 1950b) have each been shown to be powerful determiners in depth perception. It is interesting that the blur-pattern displays were not appreciably enhanced by them when divergence itself was so important. From a neural modeling point of view, this may be significant if slope-sensitive lineanalyzing units, not necessarily motion or size detectors, are adequate to account for the observers' performances.

\section{Orientation Paddle Settings}

Most of the observers were consistent at setting the paddle, but all underestimated the tilt of the simulated element plane. This coincides with the finding previously cited that subjects viewing line patterns exhibiting divergence consistently underestimate what the surface tilt would be if these were actually parallel lines in three dimensions.

\section{Practice Effect}

The results indicate that sensitivity to the divergence information in blur patterns can be increased through practice.

\section{The Role of Divergence in Orientation of a Moving Observer}

The experiments reported here have shown con- clusively that human processing of blur-pattern divergence information is adequately sensitive to make that information-as it is commonly encountered in low-level flight, automobile driving, and even walking-a potentially powerful orientation aid, at least for some observers. Relative blur-line slope or divergence can be effectively used alone for orientation; however, blur-pattern divergence is also potentially powerful when used in conjunction with other visual correlates of motion. The overall situation is extremely complex. Information about a unique location in three-dimensional space can come from the divergence pattern as it changes over time or from other aspects of the visual array such as blur-line length (which can be a concomitant of angular velocity). Other possible sources include such sensory input as that of the inner ear combined with visual eye movement feedback or the knowledge that the subject may have about such features of the array as size, shape, or orientation of the surface, and information about its texturing.

The actual utility of blur-pattern divergence will depend on the conditions of motion and of viewing. The actual dimensions that the observer does use in a particular situation obviously can be expected to to vary widely among the very large number of possible permutations of the variables in the example, and there are quite a number of other situationspecific variables that would be usable in conjunction with blur-pattern divergence.

Thus far, only absolute threshold for divergence has been considered. Even though this by itself has given valuable information about orienting potential of a rapidly moving observer, the primary reason for studying absolute divergence thresholds has been to provide a basis for investigating certain other aspects of blur-pattern divergence that may be even more influential. Studies have been completed that investigate differential thresholds for blur-pattern divergence. The divergence surface in Figure 10 shows the divergence available to a moving observer at any point on the ground and indicates that there are unique pattern divergence gradients at different parts of the field; thus, it is possible that the observer may be able to give highly refined judgments about his parameters of motion, depending on his differential threshold for divergence, by using this change of divergence over space. Ordinarily, divergence also changes over time-during altitude changes, pitch, etc. - and other work in our laboratory has shown that blur-pattern changes over time stimulate the visual system with surprising effectiveness.

In summary, these experiments have shown that high-velocity optical transformations and the facets of blur-pattern perception that accompany them belong to the general repertoire of optical transformations from which organisms are equipped to 

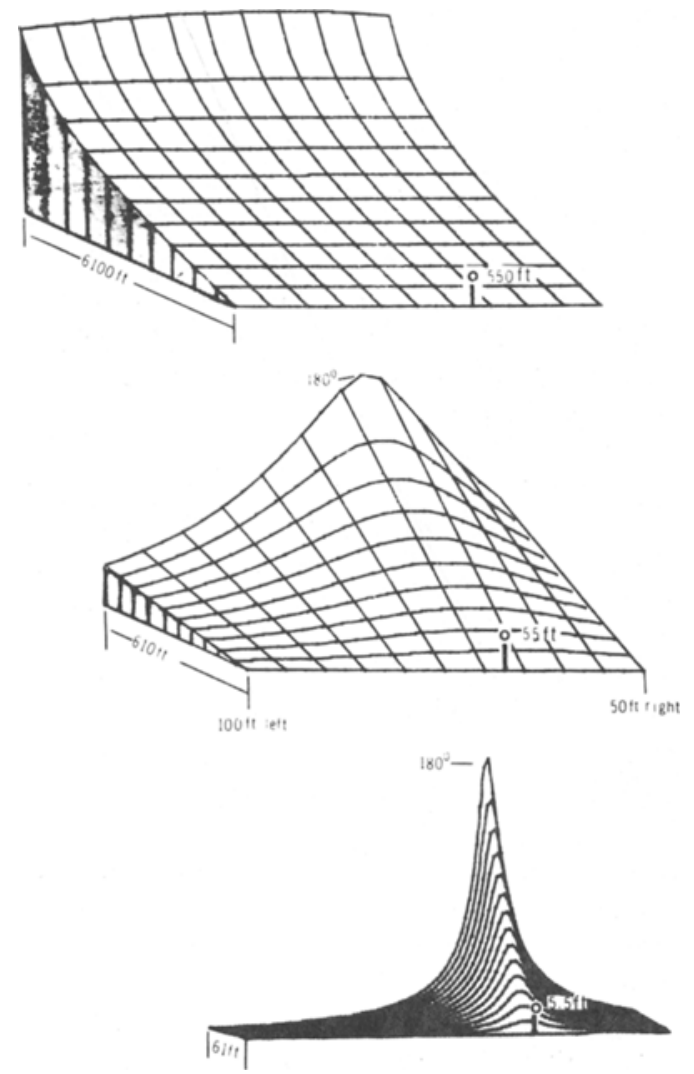

Figure 10. The blur-pattern divergences seen on the ground by the observer $(0)$ whose eye is located at the altitudes indicated. In each case, the surface extends $100 \mathrm{ft}$ to the left and $50 \mathrm{ft}$ to the right and to the front until the maximum divergence angle of $180 \mathrm{deg}$ is reached. The amount of divergence at a given point is given by the height of the surface. Surfaces have been rotated left to facilitate viewing.

select. Much work needs to be done in assessing human potential for using blur-pattern information in a wide variety of perceptual contexts and to discover under what conditions organisms ordinarily use such information.

\section{REFERENCE NOTES}

1. Marmolin, $\mathrm{H}$. The relation between perceived distance of motion in depth and amount of proximal change (Report 123). Uppsala, Sweden: Department of Psychology, University of Uppsala, 1972.

2. Von Hofsten, C. Proximal velocity as a determinant of depth (Report 139). Uppsala Sweden: Department of Psychology, University of Uppsala, 1973.

3. Harrington, T. L., \& Sprenger, D. K. Unpublished experiments, 1974

4. Michotte, A. La perception de la causalité. Louvain, Belgium: Institut Superieur de Philosophie, 1946.

5. Harrington, T. L. Unpublished observations, 1965.

6. DiOrio, M. J., \& Harrington, T. L. The dependence of divergence thresholds on temporal separation, spatial separation and retinal locus. Article in preparation.

\section{REFERENCES}

Averback, E, \& Sperling, G. Short-term storage of information in vision. In C. Cherry (Ed.), Symposium on information theory. London: Butterworth, 1961.

BECK, J. Effect of orientation and of shape similarity on perceptual grouping. Perception \& Psychophysics, 1966, 1, 300-302. (a)

BEck, J. Perceptual grouping produced by changes in orientation and shape. Science, 1966, 154, 538-540. (b)

BEck, J. Perceptual grouping produced by line figures. Perception \& Psychophysics, 1967, 2, 491-495.

Borjesson, E., \& von Hofsten, C. A vector model for perceived object rotation and translation in space. Psychological Research, 1975, 8, 209-230.

Borjesson, E., \& von Hofsten, C. Effects of different motion characteristics on perceived motion in depth. Scandinavian Journal of Psychology, 1977, 18, 203-208.

Braunstein, M. L. Depth perception in rotating dot patterns: Effects of numerosity and perspective. Journal of Experimental Psychology, 1962, 64, 415-420.

Braunstein, M. L. Sensitivity of the observer to transformations of the visual field. Journal of Experimental Psychology, 1966, 72, 683-689.

Braunstein, M. L. Rotation of dot patterns as stimuli for the perception of motion in three dimensions: The effects of numerosity and perspective. Unpublished doctoral dissertation, University of Michigan, Cornell Aeronautical Laboratory, 1960.

Braunstein, M. L. Depth perception through motion. New York: Academic Press, 1976.

Braunstein, M. L. Minimal conditions for the perception of rotary motion. Scandinavian Journal of Psychology, 1977, 18, 216-223.

Braunstein, M. L., \& Payne, J. W. Perspective and form ratio as determinants of relative slant judgments. Journal of Experimental Psychology, 1969, 81, 584-590.

Brown, J. F. The thresholds for visual movement. Psychologische Forschung, 1931, 14, 249-268. (a)

Brown, J. F. The visual perception of velocity. Psychologische Forschung, 1931, 14, 199-232. (b)

Calvert, E. S. Visual judgments in motion. Journal of the Institute of Navigation, 1954, 7, 233-251.

Cheatham, P. G. Visual perceptual latency as a function of stimulus brightness and contour shape. Journal of Experimental Psychology, 1952, 43, 369-380.

Clank, W. C., Smith, A. H., \& Rage, A. Retinal gradients of outline distortion and binocular disparity as stimuli for slant. Canadian Journal of Psychology, 1956, 10, 77-81.

Cohen, A., \& Studach, H. Eye movements while driving cars around curves. Perceptual and Motor Skills, 1977, 44, 683-689.

Cynader, M., \& Chernenko, G. Abolition of direction selectivity in the visual cortex of the cat. Science, 1976, 193, 504-505.

DeSilva, H. R. An analysis of the visual perception of movement. British Journal of Psychology, 1929, 19, 268-305.

DiOrio, M. J. Divergence threshold as a function of retinal locus. Unpublished Master's thesis, University of Nevada, Reno, 1979.

Fieandt, K. von, \& Gibson, J. J. The sensitivity of the eye to two kinds of continuous transformation of a shadow-pattern. Journal of Experimental Psychology, 1959, 57, 344-347.

Flock, H. R., \& Moscatelli, A. Variables of surface texture and accuracy of space perceptions. Perceptual Motor Skills, 1964, 19, 327-334.

Gibson, J. J. Perception of the visual world. Boston: Houghton Mifflin, 1950. (a)

Gibson, J. J. The perception of visual surfaces. American Journal of Psychology, 1950, 63, 367-384.

Gibson, J. J., \& CAREL, W. Does motion perspective independently produce the impression of a receding surface? Journal of Experimental Psychology, 1952, 44, 16-18. 
Gibson, J. J., Gibson, E. J., Smith, O. W., \& Flock, J. R. Motion parallax as a determinant of perceived depth. Journal of Experimental Psychology, 1959, 58, 40-51.

Gibson, J. J., Olum, P., \& Rosenblatt, F. Parallax and perspective during aircraft landings. American Journal of Psychology, 1955, 68, 372-386.

Gordon, D. A., \& Michaels, R. M. Static and dynamic visual fields in vehicular guidance. Highway Research Record, 1965, 84, 1-15.

Graham, C. H. Vision: III. Some neural correlations. In C. Murchison (Ed.), Handbook of general experimental psychology. Worcester, Mass: Clark University Press, 1934.

Haber, E. N., \& Hershenson, M. The psychology of visual perception. New York: Holt, Rinehart and Winston, 1973.

Harrington, T. L. Analysis of line length by the human visual system. Unpublished doctoral dissertation, University of Oregon, 1967.

Helmholtz, H. Physiological optics. New York, N.Y: Southall, 1925.

Hulbert, S. F., Burg, A., Knoll, H. A., \& Mathewson, J. H. A preliminary study of dynamic visual acuity and its effects in motorists' vision. Journal of the American Optometric Association, 1958, 29, 359-364.

ItTLESON, W. H. Size as a cue to distance: Radial motion. American Journal of Psychology, 1951, 64, 188-202.

Jansson, G. Perceived bending and stretching motions from a line of points. Scandinavian Journal of Psychology, 1977, 18, 209-215.

Jansson, G., \& Johansson, G. Visual perception of bending motion. Perception, 1973, 1, 321-325.

Jansson, G., \& Runeson, S. Perceived bending motions from a quadrangle changing form. Perception, 1977, 6, 595-600.

Johansson, G. Perception of motion and changing form. Scandinavian Journal of Psychology, 1964, 5, 181-208.

Johansson, G., \& Jansson, G. Perceived rotary motion from changes in a straight line. Perception \& Psychophysics, 1968, $4,165-170$

LEe, D., \& Lishman, R. Visual control of locomotion. Scandinavian Journal of Psychology, 1977, 18, 224-230.

LLEWELLYN, K. R. Visual guidance of locomotion. Journal of Experimental Psychology, 1971, 91, 245-261.

Ludvigh, E. The visibility of moving objects. Science, 1948, $108,63-64$

LuDvigh, E. Visual acuity while one is viewing a moving object. Archives of Ophthalmology, 1949, 42, 14-22.

Ludvigh, E. Possible role of proprioception in the extraocular muscles. Archives of Ophthalmology, 1952, 48, 436-441.

Lunvigh, E., \& Miller, J. W. Study of visual acuity during the ocular pursuit of moving test objects: Introduction. Journal of the Optical Society of America, 1958, 48, 799-802.
McDougal, W. The sensations excited by a single momentary stimulation of the eye. British Journal of Psychology, 1904-05, 1, 78-113.

Miller, J. W., \& Ludvigh, E. The effect of relative motion on visual acuity. Survey of Ophthalmology, 1962, 7, 83-116.

Olson, R. K., \& Attneave, F. What variables produce similarity grouping? American Journal of Psychology, 1970, 83, $1-21$.

Pantle, A., \& Picciano, L. A multistable movement display: Evidence for two separate motion systems in human vision. Science, 1976, 193, 502-504.

Poliock, W. T. The visibility of a target as a function of its speed of movement. Journal of Experimental Psychology, 1953, $45,449-454$.

Philitips, R. J. Stationary visual texture and the estimation of slant angle. Quarterly Journal of Experimental Psychology, 1970, 22, 289-397.

RAshbass, C. The relationship between saccadic and smooth tracking eye movements. Journal of Physiology, 1961, 159, 326-338

Schiff, W., Caviness, J., \& Gibson, J. J. Persistent fear responses in rhesus monkeys to the optical stimulus of "looming." Science, 1962, 136, 982-983.

Sмiтh, V. Scotopic and photopic functions for visual band movement. Vision Research, 1969, 9, 293-317.

Sмiтh, W. M., \& Gulick, W. L. Visual contour and movement perception. Science, 1956, 124, 316-317.

Sperling, G. The information available in brief visual presentations. Psychological Monograph, 1960, 74, 29.

Sulzer, R. L. A determination of several functions relating sensitivity of perception of parallelness to stimulus dimensions. Unpublished doctoral dissertation, Duke University, 1954.

VON DEN BRINK, G. Retinal summation and the visibility of moving objects. Utrecht, The Netherlands: H. J. Smits, 1957.

Wallach, H., \& O'CONNELl, D. N. The kinetic depth effect. Journal of Experimental Psychology, 1953, 45, 207-217.

WESTHEIMER, G. Eye movement responses to a horizontally mov. ing visual stimulus. Archives of Ophthalmology, 1954, 52, 932-941.

Whiteside, T. C., \& Samuei, C. D. Blur zone. Nature, 1970, $225,94-95$.

Yarbus, A. L. Eye movements and vision. London: Plenum Press, 1967.

Youngs, W. M. The influence of perspective and disparity cues on the perception of slant. Vision Research, 1976, 6, 97-82.

(Received for publication November 27, 1979; revision accepted May 28, 1980.) 